\title{
Towards Force Interaction Control of Biped Walking Robots
}

\author{
F.M. Silva \\ Dept. of Electronics and Telecomunications \\ University of Aveiro \\ 3810-193 Aveiro, Portugal
}

\begin{abstract}
This paper addresses the problem of modelling and control of a biped robot by combining Cartesian-based position and force control algorithms. The walking cycle is divided in two phases: single support, in which one leg is in contact with the ground and the other leg swings forward, and double support, in which the forward leg absorbs the impact and gradually accepts the robot's weight. The contact of the foot with the constrained surface is modelled through nonlinear spring-damper systems. The proposed control approach is based on simple motion goals taking into account the reaction forces between the feet and the ground. The control algorithm is tested through several experiments and its robustness is discussed.
\end{abstract}

\section{INTRODUCTION}

Many aspects of modern life involve the use of intelligent machines capable of operating under dynamic interaction with their environment. In the pursuit of solving control problems, a growing community of researchers is working towards a better understanding of machines that can balance, strike purposively and coordinate multiple degrees of freedom [1].

Walking robots exhibit complex kinematic and dynamic phenomena that make difficult their analysis and control. The major problems associated with bipedal systems are the high-order, highly coupled nonlinear dynamics and the discrete changes in the dynamics due to the nature of the walking gait. Furthermore, the degree of freedom (dof) formed between the foot and the ground surface is underactuated [2], [3] affecting the postural stability.

There has long been a dichotomy in styles used in designing and implementing biped robots. Many articles about biped locomotion focus on technological applications: Honda P2 [4], Waseda WL-12 [5] and University of Tokyo H5 [6]. In each of these robots, a fairly accurate dynamic model is used to compute dynamically admissible joint trajectories offline. These planned trajectories are then played back during walking and modified online through feedback in order to maintain stability. Other research groups use heuristic methods of control: MIT Spring-Flamingo [7] and MEL Meltran [8] are two remarkable examples. Instead of pre-computing joint trajectories, simple feedback rules are used online to control the robots. Additionally, the exploration of the specific characteristics of biped walking is the key challenge to closely relate the planning and motion control problems.

Based on the latter approach, this paper emphasis the functional properties that emerge from the interaction between the robot and its environment. This means that the competence or "intelligence" is located into the

\author{
J.A. Tenreiro Machado \\ Dept. of Electrical Engineering \\ Polytechnic Institute of Porto \\ 4200-072 Porto, Portugal
}

mechanism-environment interactions. The ground reaction forces are the key element through which new control strategies are proposed to provide the level of compliance, adaptation and dynamic stability required for walking in different contexts.

In this line of thought, the remainder of the paper is organised as follows. Section 2 describes briefly the implementation of both biped and environment models. Section 3 is dedicated to control issues and the implemented strategies. Section 4 studies the application of the proposed algorithms and presents simulation results. Section 5 concludes this paper and outlines the perspectives towards future research

\section{MODELLING OF BIPED WALKING}

\section{A. Dynamics of the Biped Robot}

The planar biped model consists of two lower limbs and an upper body (trunk and pelvis), with a total of 7 degrees of freedom (Fig. 1). The biped robot is a mechanism that repeatedly interacts with the environment through their feet. In this line of thought, the dynamic equations of motion are derived assuming the contact of both legs with the ground:

$$
\tau=H(q) \ddot{q}+c(q, \dot{q})+g(q)-J_{R}^{T} f^{R}-J_{L}^{T} f^{L}
$$

where $\tau$ is the vector of generalised torques, $q$ is the vector of joint coordinates, $H(q)$ is the inertial matrix, $c(q, \dot{q})$, is the vector of centrifugal/Coriolis torques and $g(q)$ is the vector of gravitacional torques. The transpose of the Jacobian matrices, $J_{R}^{T}$ and $J_{L}^{T}$, transforms the forces that the environment exerts on the right $\left(f^{R}\right)$ and left $\left(f^{L}\right)$ foot into joint torques.

The planar robot comprises a total mass of $M=70 \mathrm{~kg}$ and a maximum height of $L=1.4 \mathrm{~m}$ (see Table $\mathrm{I}$ ).

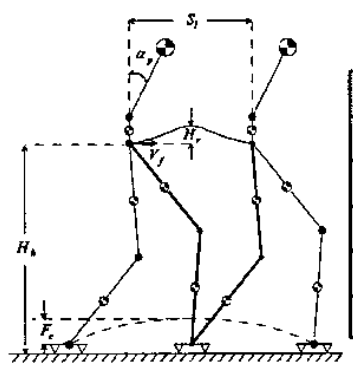

TABLE I

ROBOT LINK LENGTHS AND MASSES

\begin{tabular}{|c|c|c|}
\hline Link & Length $(\mathrm{m})$ & Mass $(\mathrm{kg})$ \\
\hline Trunk & 0.3 & 43.0 \\
\hline Pelvis & 0.1 & 2.0 \\
\hline Thigh & 0.5 & 7.5 \\
\hline Shank & 0.5 & 4.0 \\
\hline Foot & 0.24 & 1.0 \\
\hline
\end{tabular}

Figure 1. Planar biped model. 


\section{B. Environment Model and Sensorial Requirements}

The contact of the foot with the constraint surface is modelled through a nonlinear spring-damper system in the horizontal and vertical directions [9]. The tangential and normal reaction forces applied to the foot are computed as:

$$
\begin{aligned}
& f_{t}=-K_{x}\left(x-x_{0}\right)-\lambda_{x}\left(y-y_{0}\right) \dot{x} \\
& f_{n}=-K_{y}\left(y-y_{0}\right)-\lambda_{y}\left(y-y_{0}\right)^{v} \dot{y}
\end{aligned}
$$

where $K_{x}$ and $K_{y}$ are the spring stiffness, $\lambda_{x}$ is a constant, $\lambda_{y}$ and $v$ are variables that depend on the penetration depth and $\left(x_{0}, y_{0}\right)$ are the coordinates of the foot at the moment of its initial contact (see Table II).

TABLE II

\begin{tabular}{|c|c|c|}
\hline $\boldsymbol{K}_{\mathbf{z}}(\boldsymbol{N} / \dot{m})$ & $K_{y}(N / a)=$ & $\lambda_{\alpha}\left(N \Delta / m^{2}\right)$ \\
\hline $5.0 \times 10^{4}$ & $5.0 \times 10^{5}$ & $10 \times 10^{6}$ \\
\hline
\end{tabular}

ENVIRONMENT PARAMETERS.

\begin{tabular}{|c|c|c|c|c|c|}
\hline \multirow{2}{*}{} & \multicolumn{4}{|c|}{ Noum-contact, $\rightarrow$ Contact $\rightarrow$ Non-contact } \\
\cline { 2 - 6 } & Impact & Transition & Contact & Transition & Impact \\
\hline$\lambda_{y}$ & $7.5 \times 10^{8}$ & \multirow{2}{*}{$\begin{array}{c}\text { Linear } \\
\text { transition }\end{array}$} & $6.0 \times 10^{6}$ & Linear & $7.5 \times 10^{8}$ \\
& 1.5 & 1.0 & transition & 1.5 \\
\hline
\end{tabular}

It is assumed the existence of two contact points located in the extremities of the foot (at the toe and across the heel), where two force sensors are inserted (see Fig. 2).

\section{CONTROL ARCHTtECTURE}

The essence of locomotion is to transport the upper body from an initial position to a desired one throughout the action of the lower limbs. Conceptually, this goal requires the consideration of three problems: postural stability, aerial motion and contact with the ground. Moreover, it is necessary to combine, simultaneously, different control mechanisms depending on the walking phase.

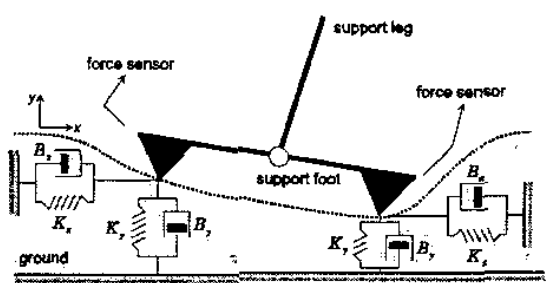

Figure 2. Environment model.

Recent advances in integrative studies of locomotion have revealed general principles and simple rules that give rise to reach behaviors. Remarkably, these high-level principles and heuristics are collective or emergent properties of biped walking arising from the interactions among the pieces on the "chessboard". Bearing this facts in mind, the locomotion process should be explored in function of two main players - robot and environment and the relations established along their natural and repetitive interactions [10]-[13].

\section{A. Hybrid Position-Force Control}

The blocks diagram of the proposed control architecture is shown in Fig. 3. Its structure takes the form of a hybrid position/force algorithm changing between force control for the $\operatorname{leg}(\mathrm{s})$ in contact with the ground and position control for the leg moving in the free space.

The motion planning is accomplished by prescribing the Cartesian trajectories of the hip and the lower extremity of the swing leg. This leg is track-controlled to place the foot on the ground with zero velocity in order to reduce the impact effects. On the other hand, when the swing foot contacts the ground, additional control efforts are used in both legs to stabilize the post-impact phase. During the double support phase the forward leg gradually accepts the robot's weight to provide for a smooth transfer of support. In what concerns the upper body, two goals should be achieved: 1) the upright stability of the pelvis; and 2) the improvement of both the dynamic stability and the forward progression regulation by exploiting the trunk's inclination.

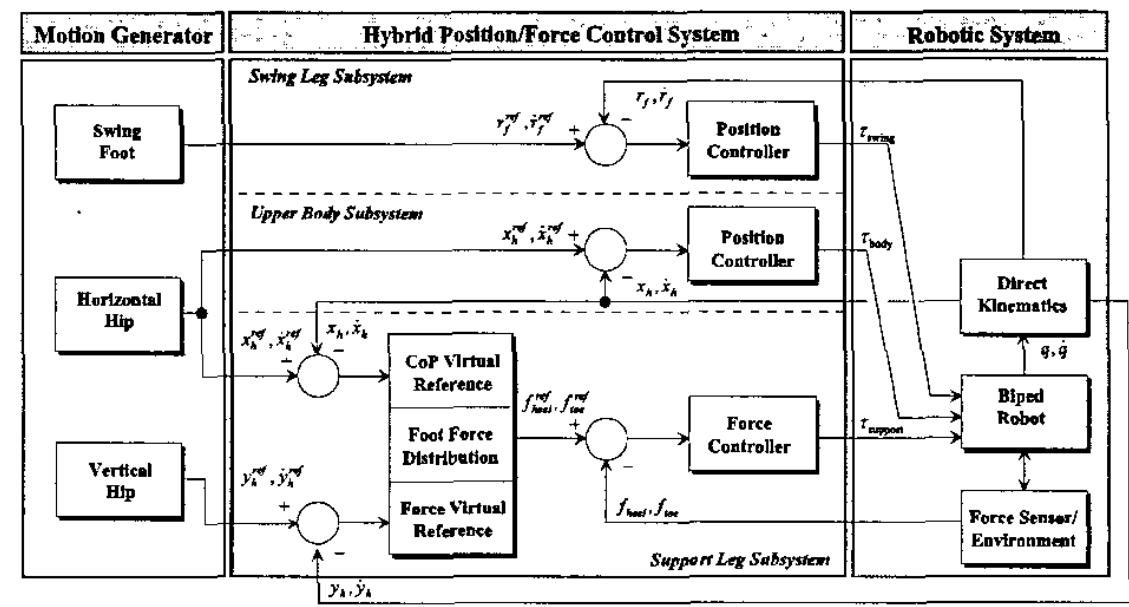

Figure 3. Goal-Oriented Force Interaction Control (GO-FIC) 


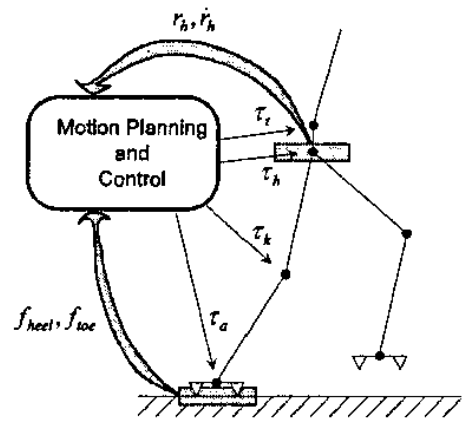

Figure 4. Basic principles of the controller.

The relevant aspects of the GO-FIC are the minimal dependence on planned variables and the consideration of the reaction forces at the feet extremities on the control algorithm. As illustrated in Fig. 4, the biped robot "feels" the forces while the controller distributes them as driving torques that regulate the desired motion of the upper body The interaction forces are the key element through which new control strategies are proposed to provide the required level of compliance, adaptation and dynamic stability. The difficult relation between motion planning and dynamic stability justifies a new line of thought: the skill of locomotion must emerges from the physical interaction between the machine and the environment itself. According to this principle, it is convenient that the controller may, in each moment, establish a relation between the desired mobility (measured at the hip) and the postural stability (measured at the ground). In this context, it is up to the degrees of freedom nearest to the ground - ankle and knee - to assure the mobility and stability of the system, and to the degrees of freedom more distant from the ground - hip and trunk - the main role of compensation.

A question that may brought up is to know how to conciliate these two imperatives - mobility and stability that in many circunstances are contradictories. On the one hand, whenever the mobility goals can be achieved, it is advantagous to maximise the energetic efficiency. On the other hand, it may be necessary to sacrifice the mobility goals (e.g., step length and/or forward velocity) to assure the dynamic stability. In this line of thought, the mobility signals are not commands but indications sent to a mechanical system possessing its own behaviour.

The rotational equilibrium of the foot is the major factor of postural instability in legged robots. Constrained forces at both feet are controlled such that stable contact is preserved between the feet and the ground:

$$
f_{\text {heel }}>0 \text { and } f_{\text {soe }}>0
$$

where $f_{\text {heel }}$ and $f_{\text {toe }}$ are the normal ground reaction forces at the toe and across the heel.

\section{B. Force Interaction Control}

The proposed control architecture enables the active steer in face of changing conditions (e.g., load or environment) by combining both force feedback with online patternmodifications. Considering the support leg, the two variables to be controlled are the normal reaction forces across the heel and at the toe, $f_{n, \text { heel }}$ and $f_{n, \text { toe }}$, respectively. In other words, the resultant normal force $f_{n}$ and the centre of pressure COP. The corresponding references are generated automatically in result of demands (motion goals) imposed to the upper body section. These are the variables that some force control law must follow. The force errors measured in each extremity of the foot can be transformed into joint torques by using the transpose of the Jacobian matrices.

The ground reaction forces combine both the gravity acting on the system and the accelerations of all body segments. In this perspective, the desired normal force is computed on-line as the sum of the robot's weight with a compensation term:

$$
f_{n}^{r e f}=B W+\left[K_{p}^{f}\left(y_{h}^{r e f}-y_{h}\right)+K_{v}^{f}\left(\dot{y}_{h}^{r e f}-\dot{y}_{h}\right)\right]
$$

Here $f_{n}^{\text {ref }}$ is the reference normal force, $B W$ is the total system's weight, $y_{h}^{r e f}$ and $y_{h}$ are the desired and real hip vertical position, $\dot{y}_{h}^{\text {ref }}$ and $\dot{y}_{h}$ are the corresponding velocities, and $K_{p}^{f}$ and $K_{v}^{f}$ are the position and velocity gains. This means that vertical errors at the hip are transformed into modifications of the reference force around its average value.

At the same time, there are different mechanisms to regulate the progression of the robot during the single support phase. One of them consists of moving directly the centre of pressure located under the foot (e.g., ankle actuation). For example, if the COP moves forward (back) the robot slows down (up) in order to remain adapted in a variety of contexts. In this line of thought, the desired location of the centre of pressure $C O P^{d}$ is obtained directly as function of the horizontal errors measured at the hip:

$$
C O P^{r e f}=K_{p}^{C O P}\left(x_{h}^{r e f}-x_{h}\right)+K_{v}^{C O P}\left(\dot{x}_{h}^{r e f}-\dot{x}_{h}\right)
$$

where $C O P^{\text {ref }}$ is the reference centre of pressure, $x_{h}^{r e f}$ and $x_{h}$ are the desired and real horizontal hip positions, $\dot{x}_{h}^{\text {ref }}$ and $\dot{x}_{h}$ are the corresponding velocities, and $K_{p}^{C O P}$ and $K_{v}^{C O P}$ are the position and velocity gains. This means that the reference COP is actively used to calculate the distribution of the total reaction force along the extremities of the support foot.

In the same way, the posterior (anterior) inclination of the body section allows the centre of mass to move forward (back) making it faster (slower) than if it remains upright. Therefore, one of the terms affecting the torque of the trunk joint, $\tau_{t}$, uses a PD control law defined by:

$$
\tau_{t}^{\text {prog }}=K_{p}^{\tau}\left(x_{h}^{\text {ref }}-x_{h}\right)+K_{v}^{\tau}\left(\dot{x}_{h}^{\text {ref }}-\dot{x}_{h}\right)
$$

where $K_{p}^{\tau}$ and $K_{v}^{\tau}$ are the position and velocity gains.

Simultaneously, the postural stability imposes limitations to the trunk motion: its inclination must remain within a limited range of the angular space and, when operating in steady state, it must converge to a limite cycle. 


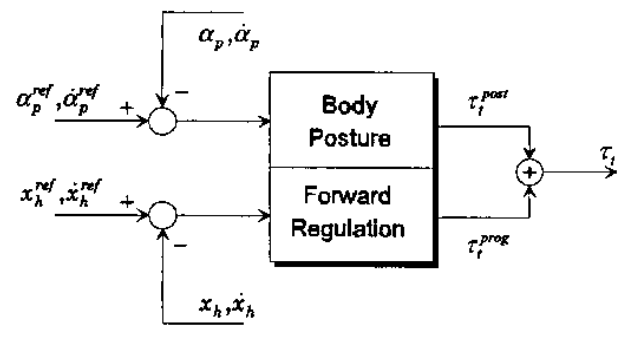

Figure 5. Control structure of the trunk section.

In this regard, the control structure of the trunk section integrates another block associated with the postural stability (Fig. 5). It is the sum of both components that actuate over the system:

$$
\tau_{t}=\tau_{t}^{\text {prog }}+\tau_{f}^{\text {post }}
$$

In what concerns the double-support (DS) phase, the question is how to solve the force distribution between legs that allows a smooth transition of support. Given the virtual references in (4) and (5), a simple method is used in which the legs gradually trade role. A linear function $\varphi(t)$ is applied in similar way to the reference forces and to the centre of pressure, that is:

$$
\begin{aligned}
& { }^{R} f_{n}^{r e f}=\varphi[B W+" \text { compensation term" }] \\
& { }^{L} f_{n}^{r e f}=(1-\varphi)[B W+\text { "compensation term"] }
\end{aligned}
$$

where the superscript " $R$ " and " $L$ " denotes the right and left legs, respectively.

Therefore, the point of application of the resultant reaction force on the rear foot moves to the middle part, making easier the next foot clearance. At the same time, the resultant reaction force on the foot placed ahead moves from the middle part, at the impact instant, satisfying the continuity of the torques.

\section{GO-FIC Controller Implementation}

This section describes the most important aspects of the controller implementation. Two dynamic selection gains $S_{R}$ and $S_{L}$ determine the instants for which force and/or position are controlled in the right and left legs, respectively. Their values depend on the particular phase of locomotion.

At each footfall the walking system suffers impact forces and incurs on additional accelerations. In order to reduce these effects, it was adopted a swing phase that minimises the impact velocities. Moreover, in the course of each stride the swing leg must flex to absorb the impact energy and, afterwards, become stiff as the support is shifted from the trailing to the leading legs. Therefore, the position/velocity references and the controller gains are modified immediately after the foot impact and the lift up occurs.

Similarly, the selection gain $S_{H}$ is responsible for the coordination of the hip joints. During the SS phase, the left hip helps to regulate the swing foot trajectory whilst the right hip assures the pelvic stability. In contrast, in the DS phase the hip actuators help together to control the upright posture of the pelvic segment.
The control laws are designed independently: the position control law consists of a PD action and the force control law consists of a PI action. It is introduced an enhancement to the PI force controller by adapting its gains during the DS phase in accordance with the distribution of forces between legs.

\section{Simulation RESUlts}

The control scheme is tested through several experiments and its effectiveness and robustness is discussed. The motion planning is accomplished by prescribing the Cartesian trajectories of the hip and lower extremity of the swing leg. In this sense, the biped motion is characterised in terms of a set of locomotion variables (refer to Fig. 1), such as step length $S_{l}$, hip height $H_{h}$, hip ripple $H_{r}$ hip pitch angle $\alpha_{p}$, foot clearance $F_{c}$ and forward velocity $V_{f}$ [14]. The next simulations are carried out assuming the locomotion variables as presented in Table III.

TABLE III

LOCOMOTION VARIABLES.

\begin{tabular}{|c|c|c|c|c|}
\hline$S_{7}(m)$ & $H_{i}(m)$ & $H_{,}(m)$ & $F_{f}(m)$ & $H_{f}(m / s)$ \\
\hline 0.4 & 0.95 & 0.07 & 0.02 & 1.0 \\
\hline
\end{tabular}

It must be pointed out that this paper emphasis steadystate locomotion. Therefore, the biped motion is simulated along several complete walking cycles in order to eliminate any transients. We assume that: $i$ ) the biped starts the movement at $t=0$ with the lift off the ground of the left foot; $i$ ) the swing foot strikes the ground at $t_{s} \approx 0.32 \mathrm{~s}$; and iii) the support transition occurs at $t=0.4 \mathrm{~s}$. This means that the duration of the DS phase is about $20 \%$ of the complete walking cycle.

\section{A. Simulation Results}

An essential demand in the study of biped locomotion is to assure the requirements for a reliable walking: $i$ ) stability of the hip height and forward velocity; ii) proper placing of the swing leg; and iii) rotational stability of the support feet.

The hybrid position/force control algorithm is derived under the assumption that two contact sensors are located at the feet. Fig. 6 illustrates the course of the vertical feet trajectories during the phases of contact and lift-up. The front leg (left) is able to absorb the impact energy avoiding any foot bouncing. Afterwards, the lift-up of the rear leg (right) occurs at an appropriate time, assuring the stability of the forward velocity.

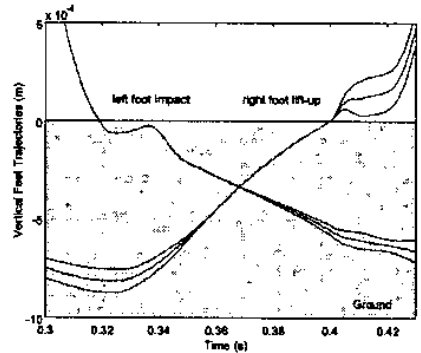

Figure 6. Vertical trajectories of the feet (hell, ankle and toe) during the impact and the lift-up phases. 


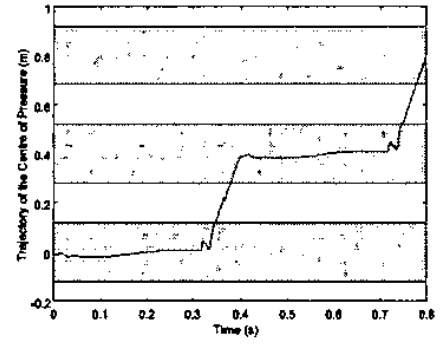

Figure 7. Temporal evolution of the global centre of pressure over two steps (the shadow boundaries denote the foot prints).

Fig. 7 represents the COP trajectory along the stride. In the SS, the COP is inside the support covered by the stance foot and then, during the DS, it moves continuously into the other foot. At the same time, the force controller is effective in accomplishing a smooth transfer of weight.

The computed joint torques are depicted in Figure 8. From these charts, we conclude that the controller is effective to regulate the impact transitions. At the same time, the application of the direct force feedback algorithm solves the force distribution problem and assures continuous torques. The simulation results of the biped walking suggest the following major comments:

1) The compensation introduced by the trunk inclination is very efficient in what concerns the dynamic stability: the stability margin rounds $85 \%$ (Fig. 7 );

2) The temporal evolution of the torques reveals the reduced value for the ankle joint (Fig. 8-a). This shows the good adaptation achieved between mobility and stability;

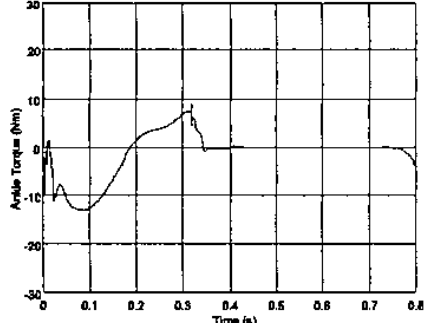

(a)

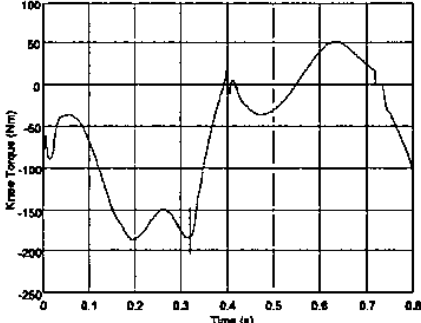

(b)
3) The temporal evolution of the normal reaction force reflects the COM accelerations. Fig. 9- $a$ resembles in large measure what results from the human locomotion;

4) Fig. 9-b and Fig. 9-c represent the joint torque and the trajectory of the phase plane reported to the trunk joint as locomotion proceeds. The major oscillations occur on the transition phases, but have no influence on the convergence to a well defined limit cycle;

In conclusion, the GO-FIC seems well-adapted to the regulation of the basic requirements presented above. The feedback of the ground reaction forces and the compensation mechanisms are efficient to generate a periodic and stable motion.

\section{B. Robustness Analysis}

One of the most common experiences to evaluate the controller's robustness is to apply an external perturbation. In this case, the perturbation corresponds to an unpredictable horizontal force $(100 \mathrm{~N})$ applied at the pelvis section, during a short period of time $(0.5 \mathrm{~ms})$. Fig. 10 emphasis the changes verified in the binary of the ankle joint and in the temporal evolution of the COP. The results obtained provide an intuitive understanding of the postural recovery: once the external force has the same direction of motion, the COP tends to move into the toe through an increase of the ankle's torque.

The second perturbation introduced corresponds to a virtual load applied on the upper trunk $(10 \mathrm{~kg})$, at a predefined instant of time $(0.2 \mathrm{~s})$. The temporal evolution of the ankle's torque (Fig. 11-a) and the global centre of pressure (Fig. 11-b) remain pratically unchanged.

Figure 8. Temporal evolution of the joint torques during a walking cycle: (a) ankle; (b) knee; and (c) hip.

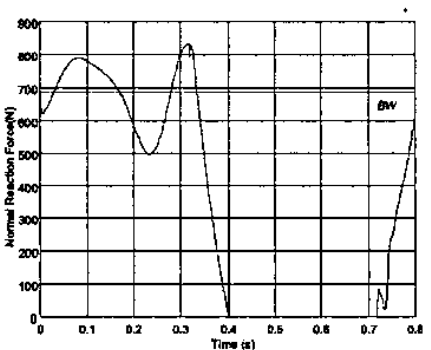

(a)

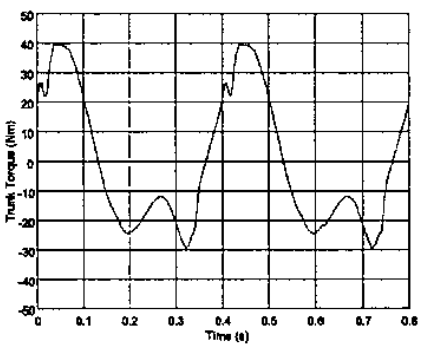

(b)

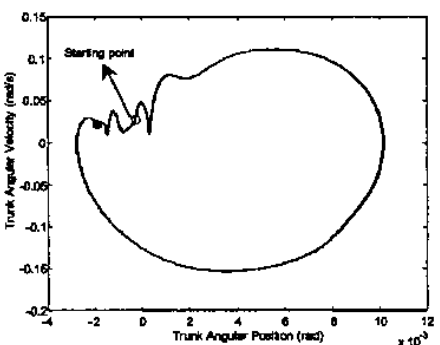

(c)

Figure 9. Temporal evolution of the: (a) normal ground reaction force; $(b)$ trunk joint torque. Phase plane: $(c)$ trunk joint. 


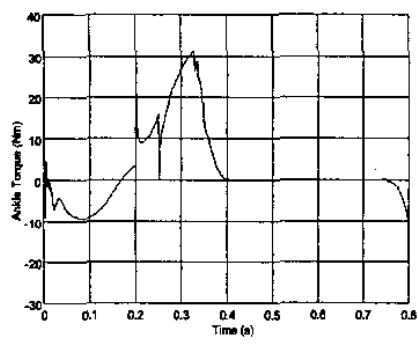

(a)

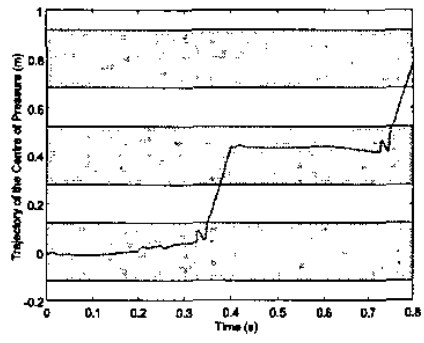

(b)

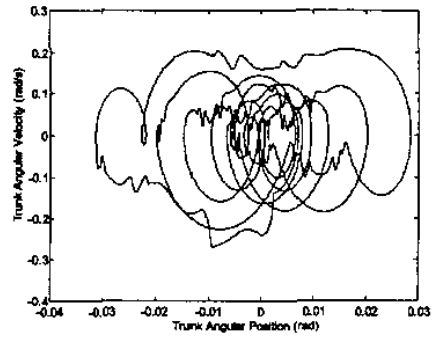

(c)

Figure 10. Temporal evolution of the: (a) ankle joint torque; (b) global centre of pressure. Phase plane: $(c)$ trunk joint.

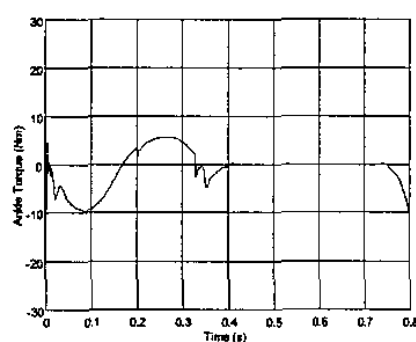

(a)

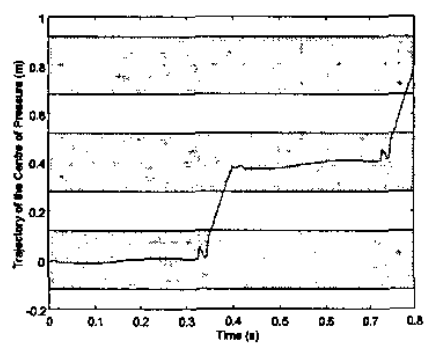

(b)

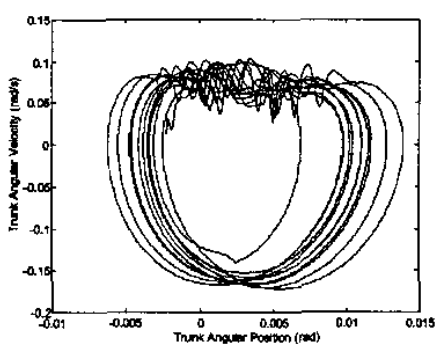

(c)

Figure 11. Temporal evolution of the: $(a)$ ankle joint torque; $(b)$ global centre of pressure. Phase plane: $(c)$ trunk joint.

Nevertheless, the posture of the trunk suffers a temporary oscillation as can be seen in the phase plane in Fig. 11-c. The automatic adaptation of the controller to load changes represents a relevant property in practical applications.

\section{CONCLUSIONS}

This paper has investigated the combination of position and force control algorithms applied to biped walking. Topics such as the algorithm robustness and postural stability were deeply discussed through several experiments. The results suggest the following major comments. First, the GO-FIC is well adapted to achieve foot stability, force compliance and different motion goals. Second, the application of the force feedback algorithm allows a smooth transfer of weight. Moreover, an adequate force distribution along the landing foot assures continuous torques. Third, the combination of position/force information results in a steady dynamic walking. However, the system's performance depends strongly on the foot trajectory, suggesting the incorporation of a compliant mechanism along with a ballistic swing trajectory.

Much work remains to be done in exploiting the potential of GO-FIC. Ongoing research focuses in two main directions: $i$ ) to apply the proposed scheme to different walking tasks; $i$ ) to extend this study to the lateral motion. A practical biped needs to be more like a human switching between different known gaits on familiar terrain and learning new gaits when presented with unknown terrains. In this sense, it seems essential to combine force control techniques with more advanced algorithms such as adaptive and learning strategies.

\section{REFERENCES}

[1] Available ontine: http://www.walking-machines.org

[2] M. Vukobratovic, B. Borovac, D. Surla, and D. Stokik, Biped Locomotion - Dynamics, Stability, Control and Application, Springer-Verlag, 1990.

[3] A. Goswami, "Postural Stability of Biped Robots and the FootRotation Indicator Point", Int. Joumal of Robotics Research, Vol.18, No.6, pp. 523-533, 1999.

[4] K. Hirai $e t$ al, "The Development of Honda Humanoid Robot", Proc. IEEE Int. Conf. on R\&A, pp. 1321-1326, 1998.

[5] J-L Yamaguchi ef al., "Development of a Bipedal Humanoid Robot Control Method of Whole Body Cooperative Dynamic Biped Walking". Froc. IEEE Int. Conf. Robotics \& Automation, pp. 368374, 1999.

[6] S. Kagami ef al., "A Fast Dynamically Equilibrated Walking Trajectory Generation Method of Humanoid Robot", Autonomous Robots, Vol. 12, pp. 71-82, 2002.

[7] J. Pratt and G. Pratt, "Intuitive Control of a Planar Bipedal Walking Robot", Proc. IEEE Int. Conf. on R\&A, pp. 2014-2021, 1998.

[8] S. Kajita, K Tani, "Experimental Study of Biped Dynamic Walking", IEEE Control Systems, vol. 16, n. 1, pp. 13-19, 1996.

[9] K. Hunt, F. Crossley, "Coefficient of Restitution Interpreted as Damping in Vibroimpact", ASME Journal of Applied Mech., pp. 440$445,1975$.

[10] S. Kawaji, K Ogasawara, J. Iimori and S. Yamada, "Compliance Control for Biped Locomotion Robot", Proc. IEEE Int. Conf. on $R \& A$, pp. 3801-3806, 1994.

[11] Y. Fujimoto, A. Kawamura, "Robust Biped Walking with Active Interaction Control between Foot and Ground", Proc. IEEE Int. Conf. on $R \& A$, pp. 2030-2035, 1998.

[12] J. Park, "Impedance Control for Biped Robot Locomotion", IEEE Trans. on Robotics \& Automation, vol. 17, n. 6, pp. 870-882, 2001.

[13] K. Loffler, M. Gienger, F. Pfeiffer, "Sensors and Control Concept of Walking Johnnie", The Imt. Journal of Robotics Research, vol. 22, n. 3-4, pp. 229-239, 2003.

[14] F. Silva, J. Tenreiro Machado, "Energy Analysis During Biped Walking", Proc. IEEE Int. Conf. Robotics and Automation, pp. 59. $64,1999$. 